\title{
Comparison of amplitude of apparent accommodation in pseudophakic eyes with that of normal accommodation in phakic eyes in various age groups
}

${ }^{1}$ Hayashi Eye Hospital, Fukuoka, Japan

${ }^{2}$ Department of Ophthalmology, School of Medicine, Fukuoka University, Fukuoka, Japan

Correspondence: K Hayashi, Hayashi Eye Hospital, 4-7-13 Hakataekimae, Hakata-Ku, Fukuoka 812-0011, Japan Tel: + 8192431 1680; Fax: + 81924415303 E-mail: hayashi-ken@ hayashi.or.jp

Received: 27 December 2004

Accepted: 15 February 2005 Published online: 8 April 2005

The authors have no proprietary interest in any of the materials described in this article
Abstract

Purpose To compare the amplitude of apparent accommodation in eyes with monofocal intraocular lenses with that of normal accommodation in the phakic fellow eyes in various age groups.

Patients and methods In all, 130 eyes of 130 patients scheduled to undergo cataract surgery, and 130 fellow eyes that had little cataract and good visual acuity of 20/33 or better were studied. The following groups were studied: in their 40s and younger $(n=20)$ or in their 50s $(n=30)$, 60s $(n=30)$, 70s $(n=30)$, and $80 \mathrm{~s}(n=20)$. Using an accommodometer (Kowa HS-9E), the accommodative amplitude was measured at 1 month after surgery.

Results In the patients in their 40s and younger or in their 50s, the amplitude of apparent accommodation was significantly less than that of normal accommodation; no significant difference was observed in the patients in their $60 \mathrm{~s}, 70 \mathrm{~s}$, or $80 \mathrm{~s}$. The incidence of patients in whom the amplitude of apparent accommodation was more than that of normal accommodation was greater in the patients in their 60s, 70s, and 80s than in the patients in their 40 s and younger and in their 50s.

Conclusion The amplitude of apparent accommodation is virtually equivalent to that of normal accommodation in patients older than 60 years of age.

Eye (2006) 20, 290-296. doi:10.1038/sj.eye.6701863; published online 8 April 2005
K Hayashi' and $\mathrm{H}$ Hayashi $^{2}$

Keywords: normal accommodation; apparent accommodation; monofocal intraocular lens; age

\section{Introduction}

Pseudophakic eyes with a monofocal intraocular lens (IOL) can achieve relatively good visual acuity in a certain range of distance; this is known as apparent accommodation. ${ }^{1-4}$ On the other hand, it is well known that the amplitude of normal accommodation in phakic eyes decreases gradually with ageing, especially from middle age on. ${ }^{5-7}$ Although we have reported previously that the amplitude of apparent accommodation also decreases with age, the change is much less pronounced than is that of normal accommodation. ${ }^{8}$ Therefore, it is conceivable that the amplitude of apparent accommodation in pseudophakic eyes becomes equivalent to that of normal accommodation in phakic eyes of older patients.

Surgeons are often perplexed as to whether or not they should extract early stage cataracts at the time of vitrectomy, penetrating keratoplasty, or glaucoma surgery. Cataract extraction does facilitate removal of the peripheral vitreous during vitrectomy, and later cataract surgery may be difficult after these surgeries.

Furthermore, surgeons also sometimes consider the extraction of an anteriorly located, pathologically large lens with little or slight cataract formation for patients with primary angle-closure glaucoma, ${ }^{9-11}$ because such surgery is effective in lowering intraocular 
pressure and in preventing an acute glaucoma attack. ${ }^{12-16}$ In these patients, the major problem due to early cataract surgery is loss of normal accommodation.

The objective of the study described herein was to compare the amplitude of apparent accommodation in eyes that underwent monofocal IOL implantation with that of normal accommodation in phakic fellow eyes of patients in various age groups. This study was performed on the assumption that the accommodative amplitude is the same in both eyes of each individual, whether the eye is phakic or pseudophakic. At ages in which the amplitude of apparent accommodation is equivalent to that of normal accommodation, early cataract extraction may be justified, even though the crystalline lens has other optical advantages over an IOL, including asphericity, ${ }^{6,17-19}$ and a blocking effect on hazardous light. $^{20}$

\section{Patients and methods}

All patients who were admitted sequentially to the Hayashi Eye Hospital for unilateral phacoemulsification surgery between January and December 2003 were screened for inclusion in this study. The original plan was to enroll 30 patients in each of five age groups: 40 s and younger (older than 20 years), and in the 50s, 60s, $70 \mathrm{~s}$, and 80s. However, since patients who met the inclusion criteria and who were in their 40 s and younger or who were in their 80s were scarce, only 20 patients could be recruited in each of these two age groups, while 30 patients were recruited in each of the other groups.

The specific inclusion criterion was that the patient was scheduled for phacoemulsification and monofocal IOL implantation in one eye and had distance visual acuity of 20/33 or better in the fellow eyes with little or slight cataract formation. Preoperative exclusion criteria were any pathology of the cornea, vitreous body, macula, or optic nerve, history of previous ocular surgery or inflammation, an irregularly shaped pupil, difficulty with follow-up, and patient refusal. Postoperative exclusion criteria were any surgical complication, including asymmetrical or out-of-the-bag IOL implantation. The study protocol was approved by the Institutional Review Board, and informed consent was obtained from each patient.

\section{Surgical technique}

One surgeon $(\mathrm{KH})$ performed all procedures using the same method that has been described previously. ${ }^{21}$ First, a $3.5-\mathrm{mm}$ scleral or $2.5-\mathrm{mm}$ clear corneal incision was made laterally for acrylic IOL implantation. All IOLs implanted were hydrophobic acrylic IOLs with a $6.0-\mathrm{mm}$ round optic and polymethylmethacrylate loops. After incision, a continuous curvilinear capsulorrhexis measuring about $5.0 \mathrm{~mm}$ in diameter was accomplished using a 25-gauge bent needle. After hydrodissection, phacoemulsification of the nucleus and aspiration of the residual cortex were carried out. Using a steel keratome, the wound was enlarged to $4.1 \mathrm{~mm}$ for hydrophobic acrylic IOL implantation. The lens capsule was inflated with sodium hyaluronate $1 \%$, after which the IOL was placed into the capsular bag. No sutures were placed in all cases. In this series, all surgeries were uneventful, and the pupils of all patients were round and showed good responsiveness to light.

\section{Main outcome measures}

The accommodative amplitude of both pseudophakic and phakic eyes was examined using an accommodometer (HS-9E; Kowa, Tokyo, Japan) at approximately 1 month after surgery. The amplitude was measured using the same method described previously. ${ }^{8}$ Briefly, the spherical and cylindrical powers in diopters (D) for best distance visual acuity (distance refraction) were first examined using an autokerato-refractometer (KR-7100; Topcon, Tokyo, Japan). The spherical equivalent value was calculated as the spherical power minus half the cylindrical power. The refractive status for best near visual acuity at $0.40 \mathrm{~m}$ (near refraction) was also examined using a near vision tester (Lumichart; HOYA, Tokyo, Japan). Then, the near point of accommodation of each eye was determined in the following manner, with the fellow eye occluded. With the spherical lens equivalent to near refraction added, the patients were asked to read a Landolt visual target, that corresponds in size to near visual acuity of $20 / 29$ at $0.40 \mathrm{~m}$. The visual target was moved slowly from about $1 \mathrm{~m}$ up to the near point at which the patients noted blurring of the target. The target was then moved back until it became clear. Measurements were repeated 10 times, and the average distance at which blurring and refocusing occurred was recorded as the near point. The accommodative amplitude was calculated by subtracting the diopters of spherical equivalent of best distance and near visual acuity from refractive power of the near point.

Visual acuity, refractive status, keratometric cylinder, and pupillary diameter were also examined at the same visit. Best-corrected distance visual acuity on decimal charts was recorded, and this visual acuity was converted to logarithm of minimal angle of resolution (logMAR) scale for statistical analyses. The keratometric cylinder in diopters was measured using the autokerato-refractometer. Pupillary diameter was measured using a Colvard pupillometer (Oasis Medical, Glendora, CA, USA). Experienced ophthalmic technicians who were unaware of the purpose of this study performed all examinations. 


\section{Statistical analysis}

Normality of data distribution was first tested using the Kolmogorov-Smirnov test. The accommodative amplitude, visual acuity, keratometric cylinder, pupillary diameter, and other continuous variables between the five age groups were compared using the Kruskal-Wallis test since the number of patients in the five groups was different. When a statistically significant difference was found, the difference between each group was further compared using the Mann-Whitney U test with the Bonferroni adjustment. The accommodative amplitude and visual acuity were correlated to the actual age of the patients using the Pearson's correlation coefficient since the amplitude showed a normal distribution. Discrete variables were compared using the $\chi^{2}$ test for independence. The difference between each group was further compared using the $\chi^{2}$ test with the Bonferroni adjustment. Any differences showing a $P$-value of less than 0.050 were considered to be statistically significant.

\section{Results}

Patient characteristics are shown in Table 1. No statistically significant differences were found between groups regarding the ratio of left to right pseudophakic eyes, keratometric cylinder in phakic eyes, and the interval between surgery and examinations. However, significant differences were found in the ratio of men and women, keratometric cylinder in the pseudophakic eyes, and pupillary diameter in both pseudophakic and phakic eyes. The mean keratometric cylinder in the pseudophakic eyes in the group younger than $40 \mathrm{~s}$ was significantly greater than that in the other age groups. The pupillary diameter decreased significantly in proportion to the age group in both pseudophakic and phakic eyes.

Figure 1 demonstrates the changes in the mean amplitude of normal accommodation in phakic eyes and of apparent accommodation in pseudophakic eyes in the five age groups. The mean amplitude of normal accommodation decreased in proportion to the age group, which was highly significantly different $(P<0.0001)$, while the difference in the amplitude of apparent accommodation was marginally significant $(P=0.0380)$. The amplitude of apparent accommodation in the group in their 40s and younger was greater than that in the group in their 70s or 80s. Furthermore, the amplitude of normal accommodation was strongly correlated with the actual age of each patient $(r=-0.758$, $P<0.0001$ ), while only a weak correlation was found between the amplitude of apparent accommodation and the actual age $(r=-0.191, P=0.0292)$.

When comparing the mean accommodative amplitude between the pseudophakic and phakic eyes (Figure 2), the mean amplitude of apparent accommodation was significantly less than that of normal accommodation in the groups in their 40s and younger and in the 50s. However, in the groups in the 60s, 70s, and 80s, no significant difference between eyes was observed in the amplitude.

Figure 3 shows the incidence of the patients in whom the amplitude of apparent accommodation in pseudophakic eyes was more than that of normal accommodation in phakic eyes. A statistically significant difference was found in the incidence between the five groups $(P<0.0001)$. The incidence of the patients in their

Table 1 Patient demographics

\begin{tabular}{|c|c|c|c|c|c|c|}
\hline & $\leq 40 \mathrm{~s}$ & $50 \mathrm{~s}$ & $60 s$ & $70 \mathrm{~s}$ & $80 s$ & P-value \\
\hline No. of patients & 20 & 30 & 30 & 30 & 20 & - \\
\hline Age & $34.4 \pm 9.8$ & $54.9 \pm 2.9$ & $64.8 \pm 2.4$ & $73.5 \pm 2.3$ & $73.5 \pm 2.3$ & \\
\hline (Range) & $(21-48)$ & $(50-59)$ & $(60-69)$ & $(70-78)$ & $(80-88)$ & - \\
\hline Gender $(\mathrm{M} / \mathrm{F})$ & $17 / 3$ & $19 / 11$ & $16 / 14$ & $8 / 22$ & $6 / 14$ & $0.0002^{*}$ \\
\hline \multicolumn{7}{|l|}{ Pseudophakic eyes } \\
\hline Left/right & $6 / 14$ & $13 / 17$ & $19 / 11$ & $12 / 18$ & $12 / 8$ & $0.1071^{\dagger}$ \\
\hline Keratometric cylinder (D) & $1.63 \pm 0.78$ & $0.96 \pm 0.97$ & $0.82 \pm 0.39$ & $0.99 \pm 0.67$ & $0.94 \pm 0.74$ & $0.0026^{*}$ \\
\hline Pupillary diameter (mm) & $5.80 \pm 0.83$ & $4.88 \pm 0.88$ & $4.67 \pm 0.80$ & $4.18 \pm 0.95$ & $4.03 \pm 0.66$ & $<0.0001^{*}$ \\
\hline \multicolumn{7}{|l|}{ Phakic eyes } \\
\hline Keratometric cylinder (D) & $0.94 \pm 0.70$ & $0.72 \pm 0.62$ & $0.78 \pm 0.35$ & $0.91 \pm 0.66$ & $1.05 \pm 0.62$ & $0.2856^{\dagger}$ \\
\hline Pupillary diameter (mm) & $5.75 \pm 0.82$ & $5.41 \pm 0.99$ & $4.92 \pm 0.82$ & $4.50 \pm 0.84$ & $4.45 \pm 0.65$ & $<0.0001^{*}$ \\
\hline Interval $^{\mathrm{a}}$ & $30.1 \pm 3.8$ & $30.5 \pm 3.2$ & $31.1 \pm 2.9$ & $30.2 \pm 2.7$ & $31.1 \pm 2.9$ & $0.6648^{\dagger}$ \\
\hline
\end{tabular}

*Statistically significant.

'No statistical significance.

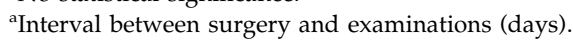

$\mathrm{D}=$ diopters 


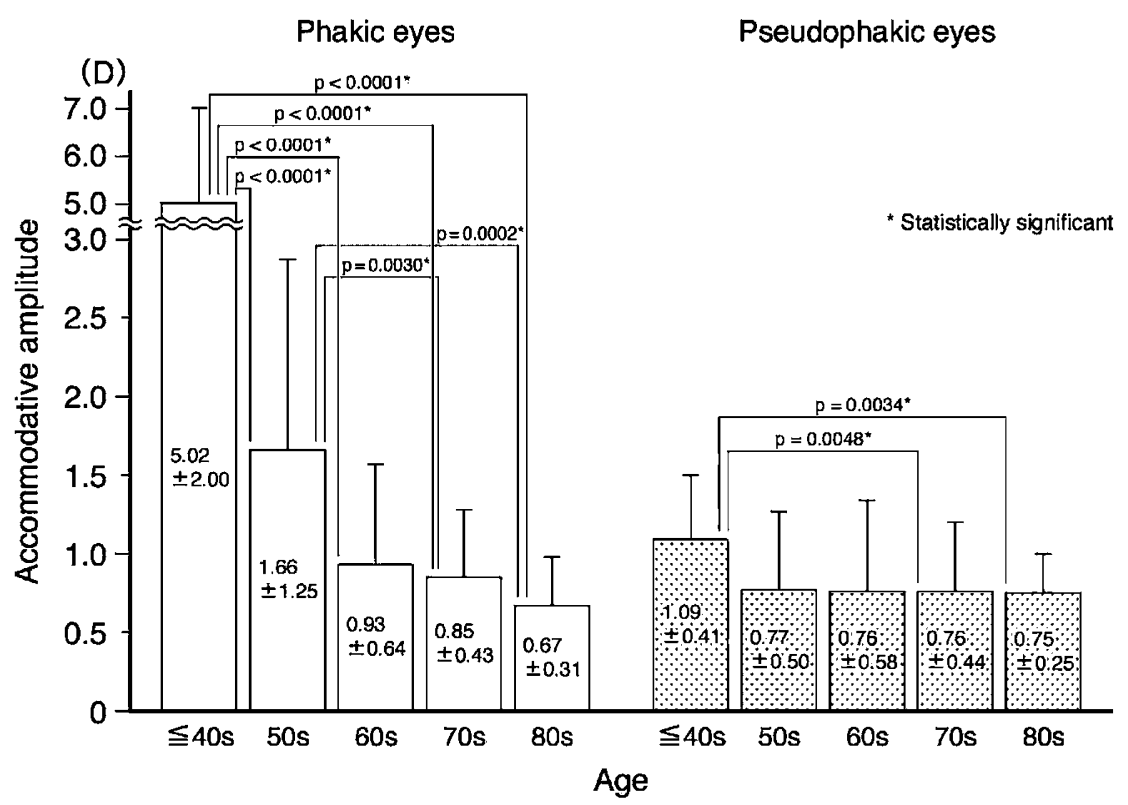

Figure 1 Changes in the mean ( \pm standard deviation (SD)) amplitude of normal accommodation in phakic eyes and of apparent accommodation in the pseudophakic eyes in the five age groups. The mean amplitude of normal accommodation decreased in proportion to the age, which was highly significantly different $(P<0.0001)$. The mean amplitude of apparent accommodation also decreased with age, but the difference was marginally significant $(P=0.0380)$. $P$-values indicate the difference between each two groups.

(D)

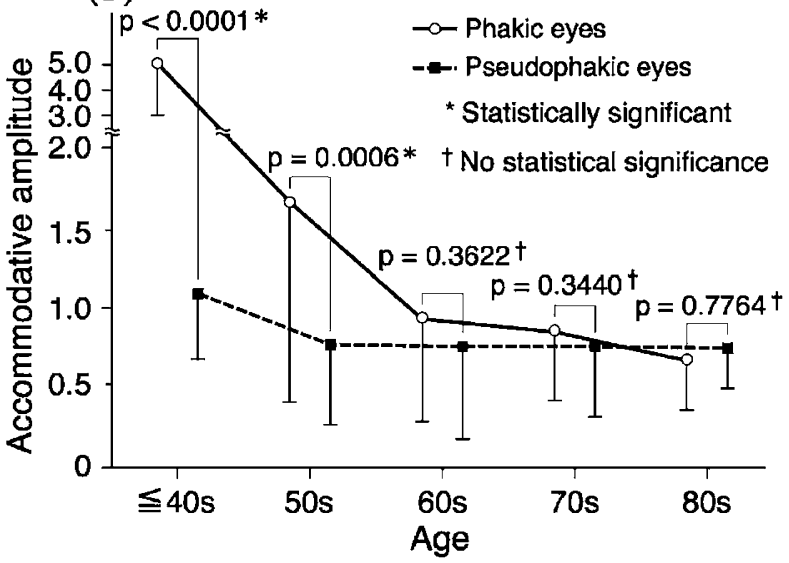

Figure 2 Comparison between the mean $( \pm S D)$ amplitude of normal accommodation in phakic eyes and that of apparent accommodation in pseudophakic eyes. The amplitude of apparent accommodation in pseudophakic eyes was significantly less than that of normal accommodation in phakic eyes of patients in their 40 s and younger and in their 50s. However, in the groups in their $60 \mathrm{~s}, 70 \mathrm{~s}$, and $80 \mathrm{~s}$, no significant difference was found in the amplitude between pseudophakic and phakic eyes.

40 s and younger was significantly less than that in the groups in their 60s, 70s, and 80s. The incidence in the group in their 50s was significantly smaller than that in the group in their 80 s.

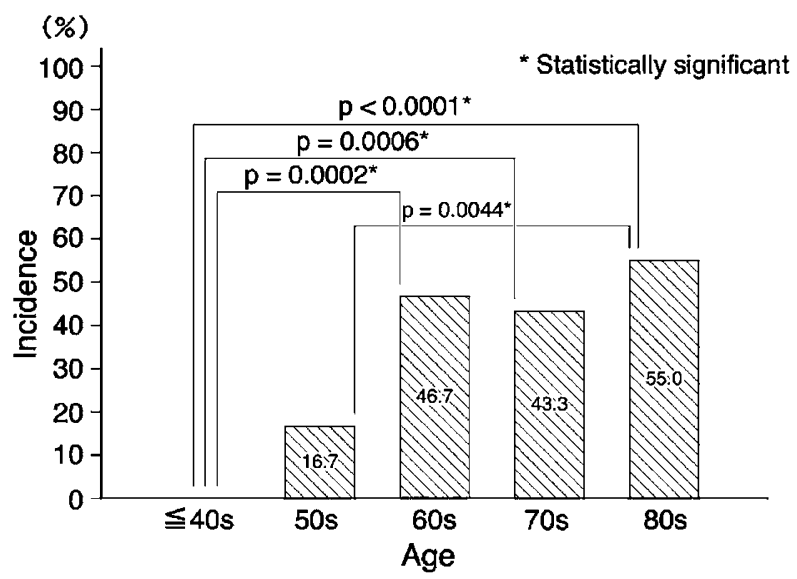

Figure 3 Incidence of patients in whom the amplitude of apparent accommodation was more than that of normal accommodation in the five age groups. The incidence of patients in whom the amplitude of apparent accommodation was greater than that of normal accommodation in the group in their 40s and younger was significantly less than that in the patients in their $60 \mathrm{~s}, 70 \mathrm{~s}$, and $80 \mathrm{~s}$. The incidence in the group in their 50s was significantly less than that of patients in their 80 s.

Figure 4 displays the mean best-corrected distance visual acuity in the five age groups. Mean visual acuity in the phakic eyes decreased significantly in proportion to age $(P<0.0001)$. Mean visual acuity in the pseudophakic eyes in the group in their 80 s was significantly worse 


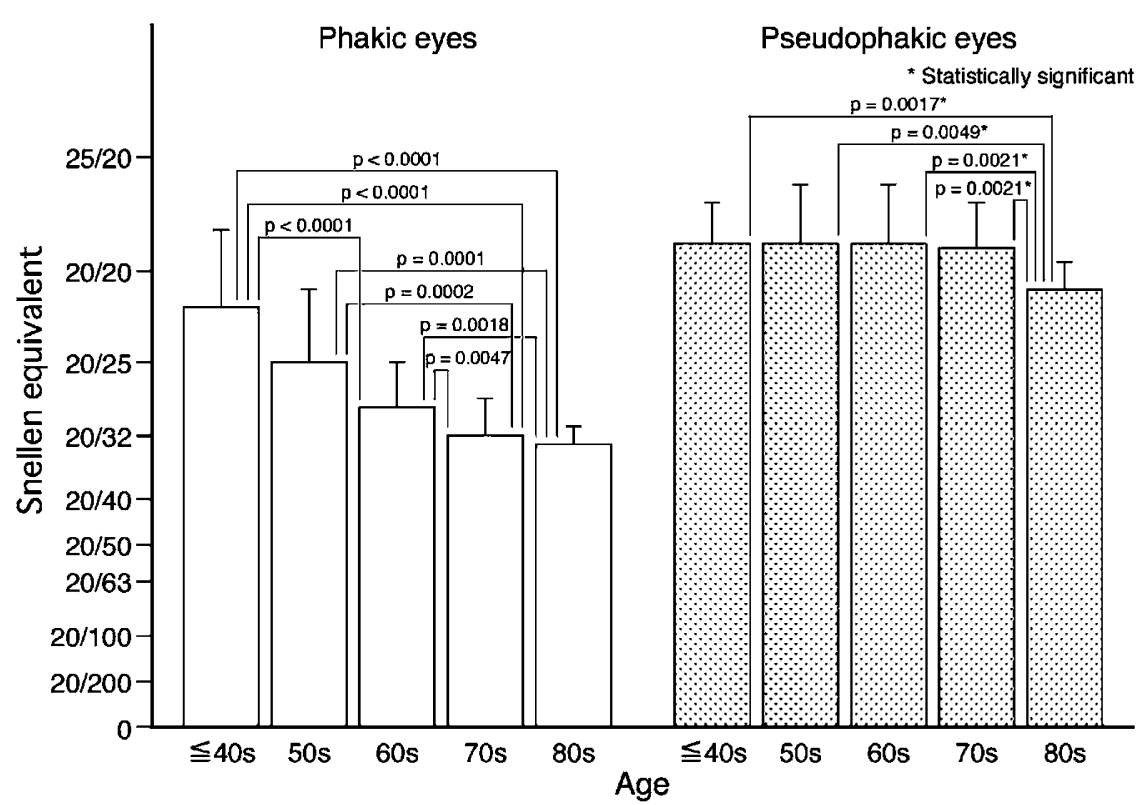

Figure 4 Mean $( \pm S D)$ best-corrected visual acuity in the phakic and pseudophakic eyes in the five age groups. Mean visual acuity in the phakic eyes decreased significantly in proportion to the age $(P<0.0001)$. Mean visual acuity in the pseudophakic eyes in the group in their 80 s was significantly worse than that in the other groups.

than that in the other groups. Furthermore, visual acuity in phakic eyes was significantly correlated to the amplitude of accommodation $(r=0.451, P<0.0001)$, while that in pseudophakic eyes was not.

\section{Discussion}

When surgeons perform vitrectomy, penetrating keratoplasty, or glaucoma surgery, they are often in a quandary as to whether or not to extract concurrently an early cataract. Furthermore, some surgeons advocate early extraction of a pathologically large lens, cataractous or not, for uncontrolled angle-closure glaucoma in order to lower the intraocular pressure $e^{9-11}$ and prevent acute angle closure. ${ }^{12-16}$ This is based on the fact that it has been reported that laser peripheral iridotomy alone is not sufficient to prevent a later rise in intraocular pressure, ${ }^{22,23}$ although controversy regarding this remains. ${ }^{24}$ The major problem of lens extraction and implantation of a monofocal IOL in patients with an early cataract is loss of normal accommodation. However, for older patients in whom the amplitude of normal accommodation has decreased to the same level as that of apparent accommodation, early cataract surgery may be justified.

Our study demonstrated that the amplitude of normal accommodation in eyes with early or little cataract formation decreased substantially in proportion to patient age. Indeed, the amplitude of normal accommodation was associated strongly with the actual age of each patient. In this series, the amplitude of apparent accommodation in eyes with a monofocal IOL also decreased with age, but the ageing decay was much less pronounced than that of normal accommodation. Consequently, the accommodative amplitude in the pseudophakic and phakic eyes became similar in older age.

The amplitude of apparent accommodation in pseudophakic eyes was less than that of normal accommodation in phakic fellow eyes of those patients in their 40s and younger and in their 50s. However, in patients in their $60 \mathrm{~s}, 70 \mathrm{~s}$, and $80 \mathrm{~s}$, there was not any significant difference in the amplitude between pseudophakic and phakic eyes.

Furthermore, when comparing the incidence of patients in whom the amplitude of apparent accommodation was greater than that of normal accommodation, the incidence in patients in their 40s and younger or in their 50s was significantly less than that in patients in their 60s, 70s, and 80s. Indeed, in the age groups of $60 \mathrm{~s}, 70 \mathrm{~s}$, and $80 \mathrm{~s}$, the incidence was virtually more than $50 \%$. These results suggest that approximately half of the patients older than 60 years of age have bilaterally equal accommodative power, even if one eye is pseudophakic.

It is known that pupillary size and astigmatism are related to the amplitude of apparent accommodation. ${ }^{2-4}$ Our result showed that the pupillary diameter of the pseudophakic eyes reduced in proportion to patient age. However, since smaller pupil diameter magnifies the 
depth of focus of the eye, ${ }^{2,3}$ a decrease in pupil diameter with age would not lead to a deterioration of apparent accommodation. In addition, our previous studies demonstrated that corneal astigmatism shifts from withthe-rule to against-the-rule astigmatism with ageing, and is the least in their $60 \mathrm{~s} .{ }^{25}$ Such changes in astigmatism is not consistent with the reduction of apparent accommodation.

We acknowledge a major limitation of this study. Although only those patients who had good visual acuity and little cataract formation were recruited, mean visual acuity in the phakic eyes worsened in proportion to increasing age. Accordingly, the decrease in amplitude of normal accommodation may be due partly to worsening of visual acuity. However, it has been reported that accommodative amplitude deteriorates with ageing even in patients with a clear lens, ${ }^{5-7}$ which is consistent with the present study. Furthermore, in practice, older patients with a unilateral cataract were very scarce, since cataract is basically an age-related condition. At any rate, the findings of the present study hold true for at least those patients in whom visual acuity becomes better than 20/ 30 with only slight cataractous change.

In conclusion, the amplitude of apparent accommodation is virtually equivalent to that of normal accommodation in the patients older than 60 years of age. Therefore, in these older individuals, simultaneous cataract surgery on eyes with only slight cataract formation may be justified during vitrectomy, keratoplasty, or glaucoma surgery, at least with regard to accommodation. Furthermore, early extraction of a pathologically large lens is also recommended for primary angle-closure glaucoma patients with early cataract. In this study, only the eyes that received a monofocal IOL were studied. However, the apparent accommodation in eyes with a multifocal IOL may be greater than that in eyes with a monofocal IOL. Further study is called for to compare the normal accommodation in phakic eyes with that of apparent accommodation in eyes with a multifocal IOL. ${ }^{26-29}$

\section{References}

1 Bettman JW. Apparent accommodation in aphakic eyes. Am J Ophthalmol 1950; 33: 921-928.

2 Huber C. Planned myopic astigmatism as a substitute for accommodation in pseudophakia. J Am Intra-ocular Implant Soc 1981; 7: 244-249.

3 Nakazawa M, Ohtsuki K. Apparent accommodation in pseudophakic eyes after implantation of posterior chamber intraocular lenses. Am J Ophthalmol 1983; 96: 435-438.

4 Nakazawa M, Ohtsuki K. Apparent accommodation in pseudophakic eyes after implantation of posterior chamber intraocular lenses: optical analysis. Invest Ophthalmol Vis Sci 1984; 25: 1458-1460.
5 Duane A. Normal values of the accommodation at all ages. J Am Med Assoc 1912; 59: 1010-1013.

6 Glasser A, Campbell MCW. Presbyopia and the optical changes in the human crystalline lens with age. Vision Res 1998; 38: 209-229.

7 Koretz JF, Kaufman PL, Neider MW, Goeckner PA. Accommodation and presbyopia in the human eye-aging of the anterior segment. Vision Res 1989; 29: 1685-1692.

8 Hayashi K, Hayashi H, Nakao F, Hayashi F. Aging changes in apparent accommodation in eyes with a monofocal intraocular lens. Am J Ophthalmol 2003; 135: 432-436.

9 Lowe RF. Aetiology of the anatomical basis for primary angle-closure glaucoma: biometrical comparisons between normal eyes and eyes with primary angle-closure glaucoma. Br J Ophthalmol 1970; 54: 161-169.

10 Lee DA, Brubaker RF, Ilstrup DM. Anterior chamber dimensions in patients with narrow angles and angle closure glaucoma. Arch Ophthalmol 1984; 102: 46-50.

11 Marchini G, Pagliarusco A, Toscano A, Tosi R, Brunelli C, Bonomi L. Ultrasound biomicroscopic and conventional ultrasonographic study of ocular dimensions in primary angle-closure glaucoma. Ophthalmology 1998; 105: 2091-2098.

12 Greve EL. Primary angle closure glaucoma: extracapsular cataract extraction or filtering procedure? Int Ophthalmol 1988; 15: 157-162.

13 Acton J, Salmon JF, Scholtz R. Extracapsular cataract extraction with posterior chamber lens implantation in primary angle-closure glaucoma. J Cataract Refract Surg 1997; 23: 930-934.

14 Hayashi K, Hayashi H, Nakao F, Hayashi F. Changes in anterior chamber angle width and depth after intraocular lens implantation in eyes with glaucoma. Ophthalmology 2000; 107: 698-703.

15 Roberts TV, Francis IC, Lertusumitkul S, Kappagoda MB, Coroneo MT. Primary phacoemulsification for uncontrolled angle-closure glaucoma. J Cataract Refract Surg 2000; 26: 1012-1016.

16 Hayashi K, Hayashi H, Nakao F, Hayashi F. Effect of cataract surgery on intraocular pressure control in glaucoma patients. J Cataract Refract Surg 2001; 27: 1779-1786.

17 Millodot M, Sivak JG. Contribution of the cornea and lens to the spherical aberration of the eye. Vision Res 1978; 19: 685-687.

18 Smith G, Cox MJ, Calver R, Garner LF. The spherical aberration of the crystalline lens of the human eye. Vision Res 2001; 41: 235-243.

19 Artal P, Berrio E, Guirao A, Piers P. Contribution of the cornea and internal surfaces to the change of ocular aberration with age. J Opt Soc Am A 2002; 19: 137-143.

20 Lehman S. Ultraviolet radiation protection. CLAO J 1985; 11: 39-45.

21 Hayashi K, Hayashi H, Nakao F, Hayashi F. Correlation between posterior capsule opacification and visual function before and after neodymium: YAG laser posterior capsulotomy. Am J Ophthalmol 2003; 136: 720-726.

22 Alsagoff Z, Aung T, Ang LPK, Chew PTK. Long-term clinical course of primary angle-closure glaucoma in an Asian population. Ophthalmology 2000; 107: 2300-2304.

23 Ang LPK, Aung T, Chew PTK. Acute primary angle closure in an Asian population: long-term outcome of the fellow eye after prophylactic laser peripheral iridotomy. Ophthalmology 2000; 107: 2092-2096. 
24 Saw SM, Gazzard G, Friedman DS. Interventions for angle-closure glaucoma: an evidence-based update. Ophthalmology 2003; 110: 1869-1878.

25 Hayashi K, Hayashi H, Hayashi F. Topographic analysis of the changes in corneal shape due to aging. Cornea 1995; 14: 527-532.

26 Steinert RF, Post Jr CT, Brint SF, Fritch CD, Hall DL, Wilder $\mathrm{LW}$ et al. A prospective, randomized, double-masked comparison of a zonal-progressive multifocal intraocular lens and a monofocal intraocular lens. Ophthalmology 1992; 99: 853-861.
27 Post Jr CT. Comparison of depth of focus and low-contrast acuities for monofocal versus multifocal intraocular lens patients at 1 year. Ophthalmology 1992; 99: 1658-1663.

28 Steinert RF, Aker BL, Trentacost DJ, Smith PJ, Tarantino N. A prospective comparative study of the AMO ARRAY zonalprogressive multifocal silicone intraocular lens and a monofocal intraocular lens. Ophthalmology 1999; 106: 1243-1255.

29 Hayashi K, Hayashi H, Nakao F, Hayashi F. Correlation between pupillary size and intraocular lens decentration and visual acuity of a zonal progressive multifocal lens and a monofocal lens. Ophthalmology 2001; 108: 2011-2017. 